Recepción: 8 de enero de 2021

Aprobación: 19 de abril de 2021

\title{
LAS PALABRAS, \\ LOS SIGNIFICADOS Y EL \\ RETORNO EN PERSONAS \\ MAYORES DE DURANGO, \\ MÉXICO
}

THE WORDS, THE MEANINGS AND THE RETURN IN THE ELDERLY OF DURANGO, MEXICO

\section{AS PALAVRAS, OS SIGNIFICADOS E O RETORNO NOS IDOSOS DE DURANGO, MÉXICO}

\section{Perla Vanessa de los Santos Amaya ${ }^{1}$}

\section{¿Cómo citar este artículo?:}

De los Santos Amaya, P.V. (2021). Las palabras, los significados y el retorno en personas mayores de Durango, México. Cultura Científica, 19, pp. 7-35.

https://doi.org/10.38017/1657463X.707

1 Doctora en Filosofía con Orientación en Trabajo Social y Políticas Comparadas de Bienestar Social. Adscrita como Profesora - Investigadora en la Facultad de Trabajo Social de la Universidad Juárez del Estado de Durango, México. Miembro del Cuerpo Académico Envejecimiento y Bienestar y de la Red Nacional de Trabajo Social Gerontológico. perla_dls@hotmail.com. https://orcid.org/0000-0002-8627-8956 


\section{RESUMEN}

En este artículo se realiza un análisis de contenido de las tramas discursivas inmersas en la cultura migratoria, específicamente aquellas palabras implicadas en el regreso a México de los connacionales mayores de 60 años que radicaban en Estados Unidos. Los datos se retomaron de entrevistas a 13 hombres y 12 mujeres de Durango (México) -elegidos por medio de una muestra intencionalque vivieron o trabajaron en el país vecino, y que contaban con experiencia de retorno en cualquiera de sus modalidades (definitivo, temporal, descanso, forzado). En el tratamiento de la información se hizo un análisis lingüístico y semántico, que en un primer momento fue cuantitativo, al retomar las frecuencias de palabras cuya repetición fue significativa. A continuación, fueron extraídos fragmentos en los que se utilizó una revisión lexicográfica que representara hechos, ideas y ambivalencias importantes para los mayores. Dentro de los principales resultados se advierte que existen arquetipos que engloban las siguientes dimensiones: espacio (territorialidad); acciones concretas que realiza (o piensa) el sujeto (morfemas verbales); y los agentes significativos (relación con los otros), cuyas palabras representan los mecanismos de tensión de las estructuras políticas y de poder en las que se vive tanto la migración como el regreso a las comunidades de origen de los sujetos. Se concluye que el Estado tiene la obligación ética de promover acciones políticas para favorecer el ejercicio de derechos humanos de los retornados.

Palabras clave: migración, retorno, palabras clave, personas mayores, lenguaje.

\section{ABSTRACT}

In this article, a content analysis of the discursive plots immersed in the migratory culture is carried out, specifically those words involved in the return to Mexico of nationals over 60 years of age who lived in the United States. The data was taken from interviews with 13 men and 12 women from Durango (Mexico) - chosen through an intentional sample - who lived and / or worked in the neighbouring country, and who had return experience in any of its modalities (definitive, temporary, rest, forced). In the treatment of the information, a linguistic and semantic analysis was carried out, which at first was quantitative, by taking up the frequencies of words whose repetition was significant. Next, fragments were extracted in which a lexicographical revision was used that will represent facts, ideas and important ambivalences for the elderly. Among the main results, it is noted that there are archetypes 
that encompass the following dimensions: space (territoriality); concrete actions that the subject performs (or thinks) (verbal morphemes); and, the significant agents (relationship with others), whose words represent the mechanisms of tension of the political and power structures in which both migration and the return to the communities of origin of the subjects are experienced. It is concluded that the State has an ethical obligation to promote political actions to favour the exercise of human rights of returnees.

Keywords: migration, return, keywords, older people, language

\section{RESUMO}

Neste artigo, é realizada uma análise de conteúdo das tramas discursivas imersas na cultura migratória, especificamente aquelas palavras envolvidas no retorno ao México de cidadãos com mais de 60 anos que viviam nos Estados Unidos. Os dados foram retirados de entrevistas com 13 homens e 12 mulheres de Durango (México) - escolhidos por meio de amostra intencional - que residiam e / ou trabalharam no país vizinho, e que tiveram experiência de retorno em qualquer uma de suas modalidades (definitiva, temporária, repouso, forçado). No tratamento das informações, foi realizada uma análise linguística e semântica, a princípio quantitativa, por meio da captação das frequências de palavras de repetição significativa. Em seguida, foram extraídos fragmentos nos quais foi utilizada uma revisão lexicográfica que representará fatos, ideias e ambivalências importantes para os idosos. Dentre os principais resultados, nota-se que existem arquétipos que abrangem as seguintes dimensões: espaço (territorialidade); ações concretas que o sujeito realiza (ou pensa) (morfemas verbais); e, os agentes significativos (relação com os outros), cujas palavras representam os mecanismos de tensão das estruturas políticas e de poder em que se vivenciam a migração e o retorno às comunidades de origem dos sujeitos. Conclui-se que o Estado tem a obrigação ética de promover ações políticas que favoreçam o exercício dos direitos humanos dos repatriados.

Palavras-chave: migração, retorno, palavras-chave, idosos, linguagem. 


\section{INTRODUCCIÓN}

La migración de México hacia Estados Unidos de América es un fenómeno que ha estado presente desde el siglo pasado. Este hecho se ha explicado desde diferentes aristas, sin embargo, mucho se ha puntualizado sobre la construcción del mercado laboral trasnacional (González González, 2009; Montaño y Herrera, 2009; Molina Foncerrada, 2009; Zúñiga y Gaspar, 2009). Estos movimientos poblacionales han sido ocasionados esencialmente por la búsqueda de mejores condiciones de vida, y ha sido un mecanismo para superar la desigualdad económica y las asimetrías que aquejan a los contextos expulsores del país. Dentro de la literatura especializada sobre migraciones se ha puntualizado reiteradamente sobre las corrientes migratorias de la región tradicionalmente expulsora. Esta región ha estado integrada por entidades del centro-occidente del país, comprendiendo Aguascalientes, Colima, Durango, Guanajuato, Jalisco, Michoacán de Ocampo, Nayarit, San Luis Potosí y Zacatecas, las cuales han mantenido una continua e ininterrumpida migración de sus habitantes de forma temporal o permanente. Cabe puntualizar que, si bien estos flujos migratorios se iniciaron a principios del siglo pasado, se agudizaron durante la puesta en marcha del Programa Braceros (1942-1964) y de la llamada Revolución verde, los cuales se desprendieron de acuerdos bilaterales para impulsar la contratación de mano de obra mexicana (Reyes y Reyes, 2016).
No obstante, a pesar de que estos programas terminaron, muchos de los contratados decidieron quedarse a vivir en territorio estadounidense. Esta situación orilló a que mexicanos sin documentación residieran en aquel país de forma ilegal, lo cual se conoce como la "era de los indocumentados”, en especial hombres que fueron empleados en sectores como la agricultura, la construcción y diversas actividades terciarias (Montes de Oca, Molina y Ávalos, 2009; Riosmena, González y Wong, 2012; Zarur, 2018; Zúñiga y Gaspar, 2009). Por lo que se refiere a las estimaciones, la Encuesta sobre Migración en la Frontera Norte revela que las personas que migraron entre 1960 y 1980 se triplicó, pasando de 760.000 a más de 2 millones de personas, y en 1900 esta cifra se incrementó a más de 4.5 millones. De forma particular, la Encuesta reporta que en el 2005 se encontraban en Estados Unidos más de 2.5 millones de personas mayores de 60 años, puesto que estos sujetos fueron los que engrosaron las franjas migratorias en tiempos anteriores, representando lo que se conoce como "envejecimiento migratorio", pues cerca del $63 \%$ ingresaron en un periodo anterior a 1975 (Reyes, 2016; Giorguli y Bautista de León, 2018; Jáuregui Díaz y Recaño Valverde, 2014). De la misma forma, datos de la Encuesta indican que para el 2016 la población de origen mexicana residente en Estados Unidos ascendió a 37.1 millones.

Hablando de forma particular de Durango, este Estado tiene una intensidad migratoria de larga data, 
sobre todo en municipios como Coneto de Comonfort, San Luis del Cordero y San Pedro del Gallo, donde el grado de intensidad migratoria es muy alto según datos del Consejo Nacional de Población. La misma fuente señala que los duranguenses se mueven a Estados como Texas, California, Illinois, Colorado y Kansas. Además, en esta entidad la captación de remesas representa para muchos de los municipios (sobre todo los rurales), la única fuente de ingresos para las familias con algún miembro migrante, lo cual evidencia la dependencia económica de estos territorios, y deja en el blanco la acentuada pobreza y las carencias sociales del contexto (rezago educativo, servicios de salud, seguridad social, espacios de vivienda y alimentación), puesto que las remesas superan en mucho el presupuesto federal asignado a estos municipios. Para el 2017, se captó un monto de más de 28.350 millones de dólares (Consejo Nacional de Población, 2010; Instituto Nacional de Estadística y Geografía [INEGI], 2015; Secretaria de Gobernación [SEGOB], 2017).

En contraparte, a pesar del pujante incremento de estos flujos, en los últimos tiempos se ha observado una disminución de estos, originados en primera instancia por el intensificado fortalecimiento e incremento de las medidas restrictivas y políticas antimigratorias ejecutadas por el Estado estadounidense. Esto se ha evidenciado como "un nuevo patrón migratorio”, que, aunque sin ser masivo por ahora, ha constituido una transformación en los movimientos poblacionales internacionales (Ávila y Tuirán, 2000; Tuirán, 2000; Zarur, 2018; Zúñiga y Gaspar, 2009). Valga decir que el regreso de mexicanos de primera y segunda generación no ha seguido una secuencia homogénea ni universal, puesto que el regreso (periódico, voluntario o forzado) ha estado condicionado por diversas razones (económicas, políticas, familiares, de salud, etc.), haciendo que no haya estimaciones precisas, sobre todo en lo que concierne a la movilidad adoptada en la población mayor (Montoya y González, 2015). Algunos datos del Instituto Nacional de Estadística y Geografía [INEGI] reportan que cerca de 35.000 mayores de 60 años regresaron a alguna entidad mexicana en el 2015. Al respecto se puede decir que el mayor número de mayores de retorno se observó en Jalisco (13.1\%), Michoacán de Ocampo (10.5\%), Baja California (8.7\%), Guanajuato (7.9\%) y Zacatecas (5.0\%); y Durango (5\%). Aunque este fenómeno es emergente, sí se aprecia que la movilidad adoptada en la vejez está teniendo diversos impactos colaterales en el ejercicio de derechos humanos, dignidad e incorporación de este conglomerado, lo cual abre desafíos en el envejecimiento trasnacional y trascultural.

Es conveniente puntualizar que este tipo de migración de mayores ha adquirido relevancia significativa dentro de los estudios demográficos, sociales, culturales y antropológico. Acercamientos como los de García Zamora y Gaspar Olvera (2016), Montoya y González 
No hay que olvidar que los retornados mayores son un grupo que durante su estancia en Estados Unidos experimentó una serie de segregaciones $y$ violencias que los hacen ser un grupo vulnerado en sus derechos humanos universales
(2015) y Reyes (2016), señalan que este tipo de migrantes se mueven hacia sus comunidades de origen por diversos motivos como: deterioro del estado de salud; reunificación familiar; apoyo de vivienda y cuidados de la salud; condiciones ambientales y espaciales favorables; y, deportación o repatriación, demandas que muchas de las veces no son resultas en las comunidades de origen. Sin embargo, no hay que olvidar que los retornados mayores son un grupo que durante su estancia en Estados Unidos experimentó una serie de segregaciones $\mathrm{y}$ violencias que los hacen ser un grupo vulnerado en sus derechos humanos universales. Por ejemplo, se habla dentro de distintos eventos como la elevada proporción de accidentes laborales, jornadas de trabajo extenuantes, falta de cobertura de seguridad social, bajo acceso a sistemas de salud (y por lo tanto obstáculos para el cuidado de la salud, diagnóstico y tratamiento), predisposición a patrones de morbi-mortalidad y sobre todo aquellos relacionados con obstáculos en la integración socio-cultural, lo que repercute en un poca estabilidad en términos de asimilación en el vecino país, y en la agudizada discriminación y exclusión a personas migrantes.

Cabe destacar que la población que cuenta con ciudadanía muestra una tendencia más envejecida (de 45 a 65 años), lo cual se relaciona con el tiempo de larga estadía en Estados Unidos, sin embargo, esta situación legal no se relaciona directamente con mejores condiciones de vida.
De acuerdo con datos censales del INEGI (2015), la principal causa de discapacidad para los migrantes de retorno es por enfermedad $(51,3 \%)$, por edad avanzada $(27,4 \%$ ) y por accidente $(18,3 \%)$. Hay que puntualizar que el $50,9 \%$ de personas mayores no está afiliado a ninguna institución de salud ni en Estados Unidos ni en México (dadas sus características de inserción a la estructura salarial). Esta situación refleja la vulnerabilidad de este sector poblacional, puesto que al no tener acceso a servicios de salud existe mayor riesgo de afectaciones a la salud, además del incremento de gastos en salud por el tipo de enfermedades que padecen las personas mayores. El 30,3\% de retornados en Durango no cuentan con acceso a servicios de salud, lo que es un desafío para las instancias estatales. Asimismo, hay que señalar que el retorno en la entidad generalmente se hace a localidades de menos de 2.500 habitantes (cerca del 30\%), lo que implica retos en cuanto a los alcances institucionales y de política pública (SEGOB, 2017).

Ahora bien, con el marcado retorno de personas envejecidas, las situaciones mencionadas traspasan las fronteras, haciendo que las situaciones de vulnerabilidad y riesgo experimentadas en etapas anteriores de vida se acumulen con las propias del contexto del retorno, lo cual deja entrever la poca capacidad del Estado mexicano para hacer frente a las demandas sociales, culturales, económicas, políticas y de salud que requieren estos sujetos. Aunque el retorno siempre se asocia a una 
posición financiera aceptable y una red familiar, también hay migrantes mayores que regresan al país con altos grados de discapacidad física o sin redes sociales y familiares (López Castro y Díaz Gómez, 2018; Lújan Ponce y Ramírez Sandoval, 2012; Riosmena, González y Wong, 2012; Zarur, 2018) (especialmente aquellas personas cuyas habilidades productivas se menoscaban y en donde aparecen cuadros de salud deteriorada, o incluso regresan sin ingreso - por pensión o jubilación-que les permita solventar sus necesidades básicas) (Giorguli y Bautista, 2018; Jaúregi y Recaño, 2014; Rivera, 2013).

En este sentido, en Durango, al igual que en el resto del país, se han impulsado acciones desde el Programa Paisano ${ }^{2}$ y algunas otras que involucran el derecho a la identidad $^{3}$ y al empleo ${ }^{4}$. Estos esfuerzos resultan ser pioneros en la atención a la población migrante, que sin duda son significativos, pero no resuelven del todo las condiciones de vulnerabilidad e incumplimiento de derechos a los que está expuesta esta población. A pesar de la importancia que tienen los migrantes

2 Este programa tiene por objetivo establecer políticas, estrategias y acciones de prevención sobre el trato digno de los connacionales que ingresan, transitan o salen de México.

3 Este ofrece apoyos para tramitar documentos oficiales en México y Estados Unidos.

4 Este programa apoya a los solicitantes a encontrar un empleo y que no tengan intenciones de emigrar nuevamente al extranjero, siendo una acción vinculante con el mercado laboral. en contextos empobrecidos y marginados, y que dejan de lado otras dimensiones como salud, vivienda, entornos seguros, educación, seguridad social, etcétera. Más aún, lo omisión de estrategias de Estado repercute en su invisibilización como sujetos de intervención pública, lo que agudiza la violencia estatal e institucional infringida hacia estas poblaciones tanto en Estados Unidos como en México.

En Durango se refleja sin duda un escenario problemático al evidenciar desafíos no resueltos en materia de aseguramiento de calidad de vida. De acuerdo con las mediciones de la pobreza del Consejo Nacional de Evaluación de la Política de Desarrollo Social, en el 2020, el $37,3 \%$ de la población en la entidad vivía alguna situación de pobreza, mientras que el $2,2 \%$ de ella se encontraba en pobreza extrema (alrededor de 40.400 personas), de manera particular el 39,4\% de personas mayores se encontraban en esta situación. Al mismo tiempo, $10,3 \%$ de duranguenses era vulnerable por ingresos, es decir, que no contaba con ingresos para satisfacer necesidades básicas, y el 27,1\% presentaba al menos una carencia (rezago educativo, servicios de salud, seguridad social, espacios de vivienda y alimentación). Retomando el coeficiente de Gini, Durango es uno de los Estados a nivel nacional con mayores condiciones de desigualdad, pues para el 2018 tenía un 0,419, escenario que reta la construcción de líneas de bienestar para los locales y para los retornados mayores de forma concreta.

\author{
Aunque el retorno \\ siempre se asocia \\ a una posición \\ financiera \\ aceptable y una \\ red familiar, \\ también hay \\ migrantes \\ mayores que \\ regresan al país \\ con altos grados \\ de discapacidad \\ física o sin \\ redes sociales $y$ \\ familiares
}


En este contexto adverso es que se presenta el retorno, como un fenómeno macrosocial, observable y empírico, y también a nivel microsocial, donde los migrantes aprehenden subjetivamente estas condiciones en las que se da la migración y el regreso a sus comunidades de origen. Estas subjetividades no son como un elemento aislado, sino como parte constituyente de las condiciones estructurales de las que son parte y producto. Así, aparecen significados desde los que se resiste, tensiona y muchas veces contradice esta experiencia vital dentro del lenguaje. Los sujetos de la migración representan estas ambivalencias a nivel cultural, económico, político y muchas veces de las relaciones de poder de las que forman parte. Entonces, ipor qué situar un análisis del retorno desde el lenguaje? Para algunos parecería fuera de lugar, sin embargo, precisamente porque la vivencia concreta nos debe aproximar a la profundidad de estas subjetividades desde las que se crean, explican y actúan en la realidad social, y desde la que se adquiere una identidad (Lozano, Peña-Marin y Abril, 2009). De esta forma, muchos son los significados atribuidos antes, en el trascurso y después del cruce fronterizo hacia Estados Unidos, marcan diferentes interdictos entre la acción, el lenguaje y el pensamiento.

Puesto en la mesa lo anterior, el artículo tiene por objetivo analizar las palabras implicadas en la decisión de retorno de personas mayores de 60 años de Durango. Tomando en consideración que este tipo de análisis lingüístico y semántico representa una aproximación a la significación que atribuyen estas personas no sólo a la migración en sí misma, sino también al retorno, el cual constituye una forma de experienciar esta movilidad social en ambos sentidos. A través de identificar cuáles son las palabras que cobran mayor relevancia para estos sujetos sociales, nos permite construir una ventana desde la cual observar las motivaciones, contradicciones, ideas y focos de interés asociados a dicho fenómeno en un contexto y espacio determinado, en este caso Durango.

\subsection{Apuntes teóricos}

\subsubsection{Construcciones sociales}

Este trabajo se sitúa en una mirada construccionista de la realidad social, entendiendo que los sujetos están inmersos en un conjunto de tramas lingüísticas que determinan su forma de entender la realidad. Esta teoría data de los trabajos de Berger y Luckmann (2008), quienes sostienen que existe una relación dialéctica entre el todo y la parte, es decir entre los sujetos y la estructura social, por lo tanto, lo que se sabe de las cosas es de naturaleza social. Para estos autores no existe una verdad absoluta de las cosas, sino que existen una multiplicidad de interpretaciones que hacen que el sujeto sea definido desde la interacción con los otros y el intercambio siempre mutuo de significados. De esta forma, Gergen (2006) menciona que para entender el estado de las cosas hay que partir de las comprensiones y 
valoraciones colectivos, las que determinan la internalización de roles y la formación de identidades del orden social del que forman parte. Luego, el carácter de la realidad es comprendido y explicado desde el sostenimiento de ese orden. Desde esta perspectiva lo que se observa del mundo es comprendido desde "artefactos sociales", producto de intercambios sociales situados históricamente que deben entenderse desde un conjunto de acciones activas y cooperativas en una comunidad sociocultural.

En el mismo sentido, López (2013) indica que el conocimiento surge de la relación existente entre los sujetos que participan de una cultura en común, y que desde sus experiencias van construyendo subjetividades insertas en el lenguaje social, que se definen no sólo por como el sujeto habla, sino también contemplan pensamientos, emociones, valoraciones y acciones. Plantea Gergen (1996) que el mundo social consiste en una serie de conversaciones que reproduce y produce. Entendiendo este argumento sobre la base de que el mundo social no es una realidad ontológica en la que estamos "depositados", sino la trama de un sistema de acciones. Otro de los autores referentes de esta teoría es Neimeyer (1999) quien sostiene que dentro de estos juegos conversacionales la identidad se produce en la relación dialéctica entre las particularidades del sujeto (lo subjetivo) y la influencia de los discursos hegemónicos en la sociedad (lo objetivo). Por lo que el autor señala que las narraciones del "yo" se refieren a los nexos entre las conversaciones con los "otros" o con lo "otro" (que, aunque aparentemente diferente, producen un determinado tipo de subjetividad) ante los cuales el "yo" se posiciona. Consecuentemente, toda expresión de uno mismo se encuentra en una relación dialógica con afirmaciones precedentes hechas por otros, al igual que por las reflexiones internas del sujeto. Entonces, las narrativas surgen de la apropiación de los discursos de los "otros" y su adaptación a los discursos objetivos del sujeto. Gergen $(1996,2006)$ señala que el sujeto incorpora la información que proviene de la cultura, confirmando, legitimando e innovando las significaciones sociales sobre lo cotidiano, de esta forma este siempre está en una reflexión constante.

\subsubsection{Lenguaje, significados y palabras}

De acuerdo con los teóricos del construccionismo social, el vehículo de la socialización más importante es el lenguaje, por lo que es el aparato legitimador de las "verdades" sociales que se objetivan dentro de los discursos que son impuestos a los miembros de la sociedad. Aunque estos autores reconocen que ciertas legitimaciones pueden requerir un grado más alto de complejidad lingüística que otras para ser comprendidas por el sujeto. Al establecer este orden, el lenguaje realiza un mundo, en el doble sentido de aprehenderlo y producirlo (Gerger, 1996). Es así que las objetivaciones del lenguaje se vuelven objetos de la consciencia individual. 
De esta manera, el hecho del mantenimiento de la realidad reside en el uso continuo del mismo lenguaje para objetivizar la experiencia biográfica. En este sentido, la cultura configura la forma de interpretar colectivamente un hecho.

En este caso, la lexicografía (que es uno de los posibles morfemas lingüísticos) se constituye como un ordenador simbólico para estructurar las relaciones con la realidad y con el propio sujeto. Las palabras siempre atribuyen un significado a las cosas, no de lo que las cosas son en sí mismas, pero sí de la interpretación que el sujeto hace de ellas de acuerdo con un espacio y momento histórico concreto, el que está mediado por la subjetividad individual y la subjetividad social (intersubjetividad) (Foucault, 2006; Nosetto, 2017). Desde esta visión el orden estructurado en el lenguaje no son hechos aislados que las personas experimentan por antonomasia, sino que son principio y fin de mecanismos en diferentes proyectos (identitarios, culturales, productivos, políticos y culturales). Consecuentemente los términos y conceptos utilizados reflejan los discursos hegemónicos del mundo social. Las palabras (que tienen atribuido un determinado significante), que se usan para narrar un hecho social, confieren atributos a este, es decir, le dan sustancia, como en el caso de este trabajo el retorno está caracterizado por una multiplicidad de características discursivas dentro de las descripciones de las personas mayores.
La migración produce, reproduce y legitima un orden en particular, y a la par que los significados de las cosas son inacabados, dinámicos y muchas veces contradictorios, también lo son los afectos, los sentires y las emociones intrincadas en este proceso (Huerta Rosas, 2008; Navarro Solano, 2007; Pérez y Lucena, 2000). Esencialmente, las palabras ordenan la realidad de la que forman parte los migrantes, haciendo que dentro de la subjetividad se les otorgue una determinada clasificación a los hechos, por lo tanto, las palabras "detienen" la realidad haciendo que se construyan categorías lógicas para darle sentido a algo. Este apartado, a la vez conversacional y a la vez conceptual, es la maquinaria desde la que el sujeto toma posición en el mundo, siempre atravesado por un sentido.

Las palabras son desde luego categorías móviles y continuas (que se sitúan en aspectos relacionales y públicos) que articulan espacios microsociales (sujeto en sí mismo) con procesos macrosociales (migración como hecho social), y que son reflejo de estructuras de poder, al mismo tiempo, entonces, hay que volver a estas tramas lingüísticas, a las circunstancias y a la relación con la estructura para entender las palabras asociadas a la migración. En sentido estricto, las palabras son un saber en sí mismo que es narrado en los juegos de lenguaje que dan cuenta de los sujetos y de los agentes significativos (lugares, cosas, instituciones, políticas, etc.) que influyen en esas narraciones desde las que se 
corporalizan en entidades particulares (Neimeyer, 1999). Razonablemente, las palabras emiten mensajes de los dispositivos de poder que contienen a las personas dentro de determinados grupos sociales, y que sostienen la afirmación de unos sujetos sobre otros (desde los que se mantiene el control y los privilegios detentados en una sociedad desigual y altamente excluyente). En contextos migratorios (tanto de idea como de regreso), las palabras definen desde lo íntimo, los atributos que rodean a la migración y que se encuentran enraizados dentro de la memoria social (Foucault, 2006; Nosetto, 2017).

Generalmente, las palabras de la migración actúan desde un doble juego: de negación y afirmación de sí mismo, dados los continuos eventos (muchas de las veces asociadas a la exclusión, discriminación y violación de derechos humanos) que definen la experiencia de la movilidad, haciéndolos vivir en un estado permanente de inseguridad dentro del habitus cotidiano. Vivir en constante incertidumbre, aunado a la precariedad laboral, bajos sueldos, las largas jornadas de trabajo y la irregularidad en el seguro de trabajo, les ocasiona determinadas narraciones del hecho social.

A la par, estar expuesto a un contexto que los rechaza, tener poca comunicación con el lugar de origen, o bien estar al tanto de las vicisitudes experimentadas por su familia les hace afrontar (y emocionalizar) esta situación de forma particular (Ariza, 2002). Estas condiciones establecen un modelamiento de la identidad del migrante, que la vive desde la subordinación y la paulatina deconstrucción de significados propios. Las palabras actúan como un efecto de panóptico desde las cuales se mira el orden social hegemónico, que en palabras de Prospero y Sieglin (2003) permiten ver la manera en que los sujetos incorporan o se resisten a ese orden. Sin embargo, estas descripciones no sólo las podemos encontrar en la experiencia de migración, sino también en el retorno, el cual es un hecho tensionado en el lenguaje.

\section{METOdOlOgía}

\subsection{Contexto del estudio}

Los resultados que se presentan constituyen una vertiente emergente del proyecto de investigación titulado "Migración de retorno, vejez y familia. Experiencias de personas mayores en Durango", financiado por la Secretaría de Educación Pública en México, el cual tenía por objetivo "Analizar la experiencia de migración de retorno y sus implicaciones sobre los vínculos familiares y sociales en personas mayores de Durango" realizado en el 20192021. Este trabajo se ubica en un enfoque cualitativo - etnográfico, ya que según lo plantean expertos en metodología de investigación estas miradas refieren posturas teóricas desde la cultura que se pretende estudiar. Este enfoque etnográfico pretende describir y analizar las ideas, significados y conocimientos de los grupos y comunidades, así que implica una interpretación profunda de sujetos que comparten rasgos culturales propios y provee 
de "retratos" de los eventos cotidianos. En esta, el investigador selecciona el lugar, detecta a los participantes, recolecta, y analiza datos intentando aproximarse a los significados compartidos, los procesos sociales y el contexto entre ellos.

Por ende, en este se recopilaron datos de 25 personas mayores de 60 años (13 hombres y 12 mujeres) en municipios (urbanos y rurales) como Durango, Canatlán, Pueblo Nuevo, Indé, Nuevo Ideal y Tepehuánes que hayan vivido o trabajado en Estados Unidos por un periodo mayor a 20 años y que cuenten con experiencia de retorno en cualquiera de sus modalidades (definitivo, temporal, descanso, forzado), conformando una muestra no probabilística intencional. Las técnicas de recolección de información fueron la entrevista a profundidad y la observación participante, las cuales fueron trascritas de forma fidedigna, cuidando el anonimato de los sujetos participantes.

\subsection{Análisis de la información}

El análisis de contenido presentado es de corte cuantitativo en un primer momento, dado que estuvo basado en la revisión de las frecuencias simples de palabras, cuya repetición dentro del corpus de los textos de las entrevistas resultó significativo. Conviene subrayar que para efectos de este trabajo solamente se tomaron los segmentos textuales que hacían alusión a la experiencia del retorno, los motivos del retorno y las emociones asociadas al retorno. A continuación, una vez extraídos estos fragmentos, se utilizó un análisis de contenido, la cual es una estrategia lexicográfica, donde se registró y revisó las palabras clave de cada categoría definida previamente, entendiendo por palabras clave aquellas cuya repetición supera la norma, y cuyo significante se asocia de forma directa con la experiencia de retorno, de modo que, una palabra clave representa ideas o hechos sociales importantes en un momento y contexto determinado (Duque, 2014; Lozano, Peña-Marin y Abril, 2009).

Estas palabras detectadas adquirieron su significado dentro del conjunto de palabras, y no de forma autónoma o aislada, es así que se valoró cada repetición de la palabra clave en el contexto lingüístico, procediendo a una fase de clasificación propia del análisis de contenido (Duque, 2014; Lozano, Peña-Marin y Abril, 2009). En este sentido, estas frecuencias sumaron a la existencia de las relaciones entre las repeticiones de la palabra y las construcciones sociales en la cultura de la migración y el retorno.

Posteriormente a la identificación de estas frecuencias se rastrearon aquellos rasgos lingüísticos que caracterizan la oralidad de los participantes. En un segundo momento se dio un proceso de categorización, el cual se distinguió por ser un acercamiento sistemático a los datos, desde el cual se construyeron nodos de contenido de las palabras gracias a la observación de rasgos comunes dentro del corpus general de contenido. Esto se realizó en el programa Atlas ti ${ }^{\circledR}$ (versión 7). Un primer barrido sobre las palabras 
clave cuya repetición y significado resultaban coherentes con el propósito del documento condujo a la clasificación en 3 ejes de análisis: territorialidades; comunicación verbal; y agentes significativos.

Dentro de estos se agruparon el conjunto de palabras seleccionadas de los testimonios de los participantes dada la importancia discursiva de determinadas unidades léxicas. Luego, estas agrupaciones se sometieron a un análisis exploratorio, principalmente lingüístico - semiestructurado. Cabe puntualizar que en este tratamiento se excluyeron aquellas palabras funcionales (de, el, la, en, los, por, y, que, del, etc.) por su poco contenido referencial y poco indicativo de las representaciones del texto, centrándonos en palabras con contenido dentro del análisis lingüístico (sufrí, dólares, duele, regresó, idea, corazón, etc.) de acuerdo con los ejes enunciados que nos evidenciaran las formas de producción comunicacional dentro de la experiencia de la migración. Es conveniente puntualizar que se cuidó que las palabras y las relaciones establecidas a partir de ellas (más que la frecuencia en sí misma), evitando que esta fuese espuria, o incluso que pudiera estar influenciado por algún tipo de limitación en el habla o discapacidad mental que pudiera afectar los resultados presentados a continuación.

\section{Resultados}

\subsection{Territorialidades}

La migración además de ser un fenómeno demográfico, social, cultural y económico, también se inserta dentro del estudio del lenguaje, desde el que se habla del sujeto que lo porta, pero también de las relaciones políticas y económicas en las que se encuentra inserto, como ya se ha mencionado. De esta forma, las narrativas también nos refieren a procesos identitarios trastocados por la trasnacionalidad, puesto que el hecho de "no ser de aquí, ni de allá" es un argumento frecuente en el acercamiento a las personas mayores que da cuenta de la fragmentación cultural que han atravesado en esta experiencia, y, por supuesto, que se evidencia al momento del regreso. Alrededor de esto, las personas mayores (considerando que la mayoría de ellas residieron en aquel país por largos periodos) tienen formas particulares de narrar sus vivencias como migrante, y que desde luego están moldeadas por dimensiones culturales, laborales y sociales e incluso de su status migratorio, que hacen que surjan discursos múltiples para entender el hecho en sí mismo.

Eventualmente, la esperanza de regresar es una expectativa que se construye social y colectivamente, y constituye una forma de mitigar las situaciones problemáticas que se viven en el trayecto migratorio, por tanto, regresar a un lugar donde se siente aparente pertenencia y seguridad es el mecanismo del que se valen los migrantes para soportar las vicisitudes de un contexto que se percibe como ajeno, y no en pocos casos marcado por la violencia y la falta de ejercicio efectivo de derechos humanos (Ramos Tovar, 2003). En este caso, 
el retorno dentro del imaginario de los migrantes representa una forma de aliviar las relaciones de poder y explotación a las que fueron sometidos durante largos plazos de su vida, que sin duda forman parte de un malestar fuertemente anclado en sus vidas. Emocionalizando el lugar de origen (territorialidad) y anclados en expectativa de un día regresar, los migrantes se refugian en este proyecto de vida a largo plazo (Osorio Campillo, Maya Sierra y Rojas Sánchez, 2015; Reyes Tovar y Martínez Ruíz, 2015).

Por añadidura, volver ocasiona que los migrantes perciban distintas disonancias del trascurrir del tiempo y el espacio. Tiempo y espacio son dos dimensiones que acontecen de forma paralela, suspendidas en las identidades de los migrantes, y que al mismo tiempo representa tener la vida en pausa: un ser que existe antes de la migración y al momento del retorno, en cuyo plano medio (vivir y trabajar en Estados Unidos) es una fase compleja, sobre todo, en condiciones donde se percibe un futuro incierto y carente de atractivo, hacen que para evitar esa sensación de miedo y ansiedad se construya el retorno como elemento vital (agudizado cuando el migrante no logra regularizar su situación legal en Estados Unidos, y que perciben el asecho constante de las políticas antimigratorias impulsadas por Estados Unidos).

En el espacio (geográfico), los sujetos construyen campos sociales que los unen con su lugar de origen, y que en muchos casos explica el retorno de migrantes durante la vejez (Reyes Tovar y Martínez Ruíz, 2015). En este caso, el retorno representa una experiencia que provoca un reacomodo simbólico y cultural que establecen con su comunidad de origen (no pocas veces complejo y problemático). Moldeándose entonces aspectos de la vida de los mayores como el arraigo, la identidad, el valor simbólico y la valoración socioemocional del espacio geográfico al momento del retorno, que muchas veces precede a la imagen "idealizada" durante su estancia en Estados Unidos (Osorio Campillo, Maya Sierra y Rojas Sánchez, 2015). Así, el territorio es una de las principales construcciones simbólicas y, por lo tanto, culturales en la subjetividad de los mayores.

Dentro de los hallazgos se advierte una relación dialéctica entre el espacio, el tiempo y la identidad de las personas mayores, generada por dos aspectos esenciales en la relación a ese desplazamiento: contexto de origen (Estados Unidos) y contexto de arribo (espacio idealizado [Durango]), estableciendo una imagen dinámica, ambivalente $y$ circulatoria. De tal manera se crean procesos de producción, creación y apropiación simbólica (unidades de significación espacial), por lo que se juegan arquetipos que definen al sujeto. Particularmente, mediante los marcadores de espacio (allá, aquí, Durango, Estados Unidos) los participantes del estudio establecieron una posición como sujetos sociales, pues de forma constante dentro de sus discursos léxicos hacían una comparación constante entre estos espacios dicotómicos y 
excluyentes aparentemente uno del otro. Dentro de los testimonios se advierten una serie de contradicciones que acarrea la experiencia migratoria. Mediante estas dos formas espaciales, tanto las representaciones pasadas como las futuras se presentan vinculadas con la situación actual.

En esta comparación entre lo que fue (vivir en Estados Unidos [Allá]) y lo que es (vivir en Durango [Aquí]), las palabras que describen vivir en Estados Unidos resultan ser polarizadas. En el caso del "Estados Unidos", las experiencias de las personas mayores indican calificativos que narran su estancia en el vecino país desde una visión del progreso y mejoramiento de las condiciones económicas y laborales concretamente, pues se observan repeticiones de sustantivos que hacen alusión a este hecho (Estados Unidos + dólares; Estados Unidos + beneficios, cheque; Estados Unidos + trabajo; Estados Unidos + dinero), lo cual representa para ellos una forma de mediar la desigualdad y las condiciones de pobreza que los aquejaban antes de ir al país vecino. Por lo tanto, esta idea de superación económica se encuentra vinculada por acciones de alta valoración y reconocimiento social, que incluso repercuten en que aparezcan emociones como el orgullo, la satisfacción y la valía de sí mismos, pues ser proveedor de la familia (traducida en el acceso a bienes materiales, forjar un patrimonio, tener ahorros, etcétera) implica haber cumplido el deber moral de los migrantes. No obstante, también se muestran las dificultades tanto culturales y laborales que trajo consigo la movilidad por medio de marcadores léxicos con clara implicación del sujeto en la acción (Estados Unidos + presionado; Estados Unidos + sufríamos; Estados Unidos + indocumentado), cuya vivencia resulta causar malestar en el tiempo que vivieron las personas en Estados Unidos, y que pone en entredicho la imagen romantizada que existe de vivir en Estados Unidos y de la idea tan difundida del "sueño americano" (tabla 1).

A pesar de las dificultades a las que se enfrentaron estas personas, reconocen y agradecen (Estados Unidos + agradezco) a Estados Unidos, como espacio, las oportunidades para el mejoramiento de sus condiciones de vida y de sus familias, pues dentro de sus narrativas se aprecian recursos lingüísticos en primera persona. Es así que dentro de las palabras clave se advierte un retorno que siempre estuvo en la mente de los migrantes, y en donde su proyecto migratorio estuvo fincado por la idea de la pensión o jubilación (Estados Unidos + pensión; Estados Unidos + jubilarme; Estados Unidos + edad), que les permitiera en un segundo momento el retorno a sus comunidades de origen, pues como lo expresaron durante sus entrevistas, que, aunque no se imaginaron llegar a la vejez, no se ven viviendo esta etapa de vida en el vecino país. 
Tabla 1. Palabras asociadas a Estados Unidos

\begin{tabular}{|l|l|}
\hline & $\begin{array}{l}\text { Acostumbrado [24], vivir [32], diferente [15], ciudad [18] dinero } \\
\text { [68], estrés [30], presión [15], presionada [16], presionado }\end{array}$ \\
$\begin{array}{l}\text { Estados Unidos } \\
\text { (allá) }\end{array}$ & $\begin{array}{l}\text { [14], trabajo [75], pensión [44], indocumentado [16], difícil } \\
\text { [10], jubilarme [18], años [24], gastan [17], caro [22], cheque } \\
\text { [35] }\end{array}$ \\
\hline
\end{tabular}

Fuente: elaboración propia con datos de las entrevistas a profundidad.

Por otro lado, las palabras para narrar el "aquí [Durango]", se muestran como el espacio enaltecido dentro de la trayectoria migrante, una construcción social que las personas mayores crearon dentro de su estancia en Estados Unidos. De tal manera que los verbos comunicacionales asumidos dentro del retorno (Durango + nací; Durango + nací, soy, querer, Durango + retiro; Durango + morir; Durango + cuidar) muestran, por un lado, sentido de pertenencia y arraigo a sus comunidades de origen (mexicano) y sus entornos familiares (casa, familia, gente), y, por el otro, la idea de encontrar bienestar al momento de su regreso (Durango + ayuda; Durango + compañía), que les asegurase en esta etapa de vida establecimiento de redes de apoyo social (tabla 2).

Otras de los sustantivos abstractos que explican el retorno de las personas mayores (Durango + edad; Durango + viejo; Durango + plan) se relacionan con el marcado paso del tiempo y el desgaste corporal. No obstante, también se reconoce que el retorno no está libre de complicaciones y añoranzas (reponiendo, reponiéndome). Una de ellas es distanciarse geográficamente de la familia que reside en Estados Unidos (extrañar, extrañaría). Asimismo, se reconoce que para el retorno es necesario contar con recursos económicos que les permitan hacer frente a sus necesidades y demandas en esta etapa de vida, pues el contexto mexicano, caracterizado por "ser pobre", hace difícil un retorno si no se cuenta con solvencia económica para hacer frente a esta movilidad en la vejez. Así, además del costo económico del retorno del migrante deben asumirse los costos afectivos del retorno, tales como la separación de la pareja e hijos residentes en EUA, la viudez, la depresión, la deportación (que es vista como fracaso), la pérdida de trabajo, la soledad, el impacto emocional de los hijos (los que muchas veces han migrado también y que se encuentran indocumentados) y la llegada a un espacio donde las redes de apoyo social son limitadas (García Zamora y Gaspar Olmera, 2016; González González, 2009; Montaño de la Concha y Herrera Lasso, 2009; Rivera Sánchez, 2013).

De tal manera que el retorno a su lugar de origen se convierte en una nueva migración, proceso que implica para las personas un proceso con niveles de estrés que llegan a superar la capacidad de adaptación de este, pues él ha cambiado, su familia y sus respectivas comunidades de origen. Para Sobrino (2013), 
el reencuentro con lo dejado se convierte en algo agotador, cíclico y paradójico, que revive el duelo de la inmigración y la necesidad de volver a elaborarlo, avivando muchas de las ocasiones la falta de vinculación y el aislamiento social. Estas experiencias se vuelven significativas, pues por una parte el sujeto se mueve hacia un futuro lleno de sentido (regreso), pero, por otro, lo orilla a estar procesando duelos constantes que desembocan en cambios en su posición en el mundo e identidad (Neimeyer, 1999).

En ese sentido, también habría que puntualizar sobre las características de los territorios de arribo de los retornados (como el de Durango caracterizado por situaciones de pobreza y falta de políticas de apoyo a los migrantes) hacen que su reincorporación en la etapa de la vejez se vuelva problemática, sobre todo porque estos se encuentran en una etapa de vida que los hace demandar ciertos satisfactores de bienestar en materia de salud, seguridad social, ingreso y cuidado no resueltos para la población en general. Por lo tanto, el regreso de este tipo especial de migrantes tiene que visualizarse sí como un reto a nivel privado, es decir de las familias y la comunidad, pero también debe incorporarse como un asunto de interés político y público.

Tabla 2. Palabras asociadas al contexto de retorno

\begin{tabular}{|l|l|}
\hline $\begin{array}{l}\text { Durango } \\
\text { acá) }\end{array}$ & $\begin{array}{l}\text { Rancho [32], mejor [28], soy [40], mexicano [28], casa [24], } \\
\text { familia [47], viejo [32], plan [18], querer [37], queriendo [18], } \\
\text { venir [31], venirse [28], verlos [34], hijos [30], extrañaría [20], } \\
\text { disfrutar [32], antes [42], apoyo [31], ayuda [35] }\end{array}$ \\
\hline
\end{tabular}

Fuente: elaboración propia con datos de las entrevistas a profundidad.

\subsection{Comunicación verbal}

La identidad trasnacional de los mayores se encuentra tensionada directa $o$ indirectamente con los verbos que utilizan los retornados mayores para describir sus motivaciones para regresar a Durango, y que son producto y reflejo de la estructura social. Dentro del corpus de interés para esta dimensión se encuentran aquellas repeticiones verbales que de forma recurrente mencionaron los sujetos de estudio, que desde luego son acciones dentro del sentido y significado colectivo, y sin duda también dentro de los contextos lingüísticos en los que aparecen estas palabras articuladoras del pensamiento, la acción y la valoración. En los morfemas verbales pudimos observar diversos verbos implicados a la experiencia migratoria: instrumentales (hacer, ir, trabajar y tener; vivir), expectativa (estar, querer,); cognitivos (saber, ser); y expresión (decir).

En los primeros, los verbos instrumentales, la idea de ir a Estados Unidos se asume como un hecho para mejorar la vida, sin embargo, también se observa que los participantes no tenían la intención de quedarse a vivir allá (Vivir + temporal; Vivir + años; Vivir + esperar), 
pues los sustantivos abstractos hacen alusión a una idea de retorno (Ir + México; Ir + Durango; Ir + rancho), de tal forma, las formas verbales adjuntas a esta idea de ir ( $\mathrm{Ir}+$ vida; Ir + familia) reafirman la idea de querer regresar al país después de lograr ahorrar y tener un patrimonio para la vejez, la idea progresista que se ha señalado (tabla 3 ).

En el verbo de trabajar, es una misión que emprenden muchos de nuestros connacionales para mejorar las condiciones de vida, y que, en la posición de las personas mayores, se asume como una etapa para lograr el progreso. A pesar de esto, con el advenimiento de los años y la vejez, el cansancio se hace presente por las agotadoras jornadas a las que han sido expuestos en el vecino país y las condiciones adversas a las que se enfrentan en Estados Unidos, por ello, vinculados a la forma verbal del trabajo, como categoría analítica que define al sujeto, se encuentran la idea de jubilación, retiro, pensión y seguro, privilegio que es alcanzado por pocos migrantes, dada su condición migratoria (en especial aquellos que trabajaron con seguros irregulares y que su posición laboral es marginal). Vivir en Estados Unidos se vive de forma transitoria y siempre en un estado constante de espera para poder regresar a su lugar natal (Vivir + temporal; Vivir + esperar; Vivir + años). Junto con ello se aprecia la construcción del retorno como una situación futura y deseable dentro de los discursos de los participantes, cuya argumentación arguye a que el retorno se da en una aparente elección por voluntad y en condiciones propicias para el regreso a sus comunidades de origen, por lo tanto, se asume que esta nueva migración se relaciona con la capacidad personal de controlar su propio futuro, no obstante este está condicionado por aspectos estructurales y económicos que entraman las decisiones de los sujetos y que no pueden pasar desapercibidas al momento de que las personas mayores deciden emprender el viaje a Durango, ya sea de forma temporal, definitiva o forzada.

Tabla 3. Verbos comunicacionales instrumentales

\begin{tabular}{|l|l|}
\hline Hacer & Dolaritos [61], dólares [75], casa [54] \\
\hline Ir & $\begin{array}{l}\text { Dólares [75], dinero [30], vida [43], familia [71], México [47], Durango } \\
\text { [39], rancho [28] }\end{array}$ \\
\hline Tener & $\begin{array}{l}\text { Tuve [38], tenía [41], teníamos [29], idea [67], allá [101], pensión [41], } \\
\text { ayuda [71], papeles [47] }\end{array}$ \\
\hline Trabajar & $\begin{array}{l}\text { Jubiles [101], jubilar [45], retirar [39], pensionar [47], pesado [49], } \\
\text { cansado [38], }\end{array}$ \\
\hline Vivir & Temporal [58], años [21], esperar [47] \\
\hline
\end{tabular}

Fuente: elaboración propia.

En las entrevistas se pudo constatar la visión como sujetos "activos", y que, desde estas aspiraciones de progreso, el retorno es la continuidad de sus trayectos vitales. De tal suerte que, en los procesos 
de comunicación, las formas verbales de expectativa, específicamente de tener, se representa como el logro de beneficios materiales y de seguridad social que obtienen ese privilegio. También se encuentran plasmadas dentro de las palabras encontradas algunas de las vicisitudes a las que se enfrentan como el vacío, el temor y el miedo constante, sobre todo si se piensan asediados por procesos vejatorios y que ponen en entredicho su dignidad como personas. Aunque también reconocen en ese sentido el gusto que les provocó estar en Estados Unidos, también se encuentran relacionados aspectos de inseguridad, soledad y poca estabilidad económica, emocional y familiar. Razonablemente, pensar en un retorno que los haga reunificarse con la familia de origen es algo que siempre tuvieron en mente estas personas, pues como lo comentan dentro de los fragmentos, allá existe poca disposición de tiempo, familia y cuidado en algún momento de necesidad, ya que muchos de sus hijos trabajan largas jornadas, dejando poco espacio para la atención, convivencia y cuidado en el contexto receptor.

Tabla 4. Verbos comunicacionales de expectativa

\begin{tabular}{|l|l|}
\hline Estar & $\begin{array}{l}\text { Estados Unidos [84], gabacho [41], gustó [35], compañía [39], gastos } \\
\text { [47], familia [71] }\end{array}$ \\
\hline Querer & Visitar [43], hijos [31], fecha [29] \\
\hline
\end{tabular}

Fuente: elaboración propia.

Por lo tanto, no es difícil asumir en estas circunstancias verbos cognitivos como el saber y el ser está formado por verbos en modo subjuntivo y sustantivos abstractos relacionados con sus deseos, estas características se localizan en una idea de una situación menos perturbadora. Estas palabras clave articulan un objetivo a largo plazo. De tal forma que dos procesos relacionales "somos" y "estamos" se utilizan para la evaluación que hacen los participantes de sus condiciones de vida. El retorno se vive como un conocimiento que se sabe, muchas veces desde que entra al país de destino, y que concluido el proyecto migratorio (consciente e inconsciente) se asume el regreso. De tal suerte, que ser un retornado vincula determinadas formas emocionales como la felicidad, la tranquilidad, el encantamiento y la tristeza, lo cual deja entrever que el retorno también se vive de forma compleja, sobre todo si no hay satisfactores de bienestar en el contexto duranguense. Estos son campos semánticos que expresan la voluntad de querer regresar, al igual que esa intención de persuadirse a sí mismos ante el proyecto migratorio. En otro aspecto, los verbos comunicacionales de expresión "decir", se relacionan con la decisión de regresar, que como se muestra en las palabras vinculadas, fue una situación pensada, aludiendo a mejorar en relación con el estilo de vida tenido en Estados Unidos (Decir + decisión). 
Tabla 5. Verbos comunicacionales cognitivos

\begin{tabular}{|l|l|}
\hline Decir & Mejor [52], decidimos [35], decidí [41], decisión [64] \\
\hline Saber & $\begin{array}{l}\text { Esperar [44], años [21], vejez [54], entrar [40], edad [48], aterra [6], } \\
\text { estarme [27], familiares [47] }\end{array}$ \\
\hline Ser & $\begin{array}{l}\text { Feliz [15], tranquilo [34], acompañado [45], indocumentado [62], } \\
\text { residente [32], visa [44], papeles [65] }\end{array}$ \\
\hline
\end{tabular}

Fuente: elaboración propia.

\subsection{Agentes significativos}

Al igual que la cuestión territorial y comunicativa planteada con anticipación, dentro del retorno se juegan diversos aspectos de la construcción identitaria. Estos tienen que ver con las relaciones interculturales, la diversidad y la producción que se hace de lo "otro" en la experiencia de la migración (Boivin, Rosato y Arribas, 1999; Jofré, 2001; Salas, 2012). De esta manera, el fenómeno de la migración produce el encuentro o desencuentro de prácticas y significados culturales desde las que los sujetos moldean "lo otro". Los estudios advierten que la construcción del otro supone siempre una relación de negación y complementariedad de la identidad. Así, el otro ayuda al sujeto a afirmar la propia personalidad del sujeto, por lo que, su posición en el mundo está determinado por la existencia de los "otros" (Bauman, 2007). Sobre todo, en el caso de mayores que radicaron en Estados Unidos por largos periodos, han estado expuestos a una serie de modificaciones en su entorno individual y social, detentando múltiples producciones de lo "otro". Lo anterior tiene implicancias desde luego en la posición que el sujeto da a sí mismo a la propia existencia y a la de los demás (Bauman, 2007). De tal manera que en los "retratos" que se hacen en ambos lados de esta dualidad se encuentran insertos objetos, sujetos y prácticas al interior de la cultura migrante (Foucault, 1990). Estas miradas sobre el otro es una mirada en primer lugar sobre la propia identidad de quien la hace, de tal suerte que no es posible mirar al otro sin antes analizar la mirada de quien observa: el otro constituye, posiciona y cuestiona al propio sujeto.

Entonces en la construcción de los sujetos y agentes significativos dentro del retorno hay una forma de autoidentificación, jugándose la cuestión del reconocimiento de sí mismo en este proceso, y que interfieren de forma directa en la decisión de regresar de las personas mayores. En este caso, aparece "Dios" como agente trascendental que da sentido a la vida de estos sujetos, lo cual resulta ser congruente con la importancia que le otorgan las personas migrantes a la religión, ya que esta es un mecanismo de contención en situaciones catastróficas o de crisis por las que atraviesan dentro de la experiencia migratoria, y por supuesto, también en el retorno. A partir de esto, esta contención se realiza 
frente a condiciones estructurales que son adversas para los migrantes, tanto en Estados Unidos como en sus comunidades de origen, y que es un recurso ante la violencia, la soledad, la pobreza y demás situaciones por las que han atravesado los migrantes. En las palabras vinculadas a este ente abstracto se observa un acompañamiento espiritual y agradecimiento por los beneficios materiales alcanzados (camino, vuelta, permitió, permite) en el contexto migratorio, así como en el contexto de regreso (Dios + santo; Dios + vuelta; Dios + camino). Asimismo, se distingue que "Dios" representa una forma de explicarse a sí mismos y explicarse la realidad que los rodea.

Tabla 6. Agentes trascendentales

\begin{tabular}{|l|l|}
\hline \multirow{2}{*}{ Dios } & $\begin{array}{l}\text { Bueno [32], mejor [47], padre [22], permitió [25], permite [35], } \\
\text { santo [29], quedó [21], quedarme [31], vuelta [18], camino } \\
{[32]}\end{array}$ \\
\hline
\end{tabular}

Fuente: elaboración propia.

Por otro lado, el retorno del mexicano modifica la estructura etaria del país, de las comunidades a las que retornan, y en especial modifican la dinámica de los hogares y los arreglos y estrategias familiares. En las relaciones familiares y personales, el proceso de reajuste a otra dinámica social en un contexto (ahora contexto de retorno) sin duda ha cambiado, no obstante, que el migrante haya salido de ese mismo sitio (López Castro y Díaz Gómez, 2018). En este sentido, las palabras apuntan a sentimientos de arraigo, pertenencia y valoración social de la familia, que según los discursos proporcionan seguridad, apoyo mutuo, solidaridad y proximidad (tanto como comunitarios, sociales y familiares). En lo que se refiere a la vinculación familiar aparece como palabras esposo (a), hijos (as) y familia, en los que se hace alusión a un estado de separación y pérdida tras la migración, pero que, en el retorno se espera poder reunificarse, recibir apoyo y cuidados.
Desde luego la aparición de estos agentes significativos deviene de procesos de construcción de "lo otro", produciendo expectativas idealizadas al momento del regreso, que no siempre se cumplen a cabalidad.

En los sustantivos abstractos de estas dimensiones (Familia + separados; Familia + esperanza; Familia + juntarme) y en los morfemas verbales (venir, separar, perder, juntar, extrañar) se aprecia el deseo de estar en familia una vez concluida la etapa laboral. Entonces, la familia emerge como espacio preferencial donde se vivencian con mayor intensidad las relaciones humanas que han sido trastocadas por los efectos de los flujos migratorios (Ribeiro, 2002). A partir de los procesos migratorios se configura la familia que permite evidenciar nuevas formas de relación y vínculos que se establecen entre los migrantes y su familia ubicada en el país de origen, por medio del estudio de los 
procesos familiares. Concretamente la fragmentación de vínculos, reunificación y desintegración familiar, se puede evidenciar diversas formas de gestionar la familia en contextos de alta migración (Ariza, 2002; Lujan y Ramírez, 2012) (ver tabla 7).

Las narrativas de los participantes indican que regresar está en gran medida influenciado por la idea de compensar el tiempo de ausencia, tanto con la esposa (o) y con los hijos, sobre todo por el carácter de amor filial que se juega en los vínculos de parentesco, lo cual da a los sujetos sentimientos de acompañamiento, ayuda mutua y cuidado en situaciones de crisis o de salud. No obstante, el precio de la reunificación con la familia de origen tiene sus costos (emocionales, sociales y subjetivos), al construir lazos que trascienden las fronteras nacionales, creando vínculos y afectos en ambos países, por lo tanto, tienen que asumirse nuevas dinámicas y arreglos familiares que suelen causar malestar a los retornados, y que hacen que surjan emociones diversas. Aunque los mayores estén en posibilidad de viajar a Estados Unidos para poder visitar a sus familiares, también se indican situaciones que dificultan el traslado, sobre todo las condiciones de salud y dependencia con que regresan. No menos importante es señalar que la red familiar (nacional o trasnacional) aparece como un espacio de contención e integración para las personas mayores, representando una gran paradoja, pues se construye dentro de lo "otro", pero que en lo real no cumple con la expectativa que se tiene.

Tabla 7. Agentes familiares

\begin{tabular}{|l|l|}
\hline Esposo (a) & $\begin{array}{l}\text { Señor [67], señora [41], años [21], viniera [35], venir [28], fui [47], } \\
\text { separar [34], separados [28], perdí [28] }\end{array}$ \\
\hline Familia & $\begin{array}{l}\text { Siempre [42], estar [35], juntarme [39], estar [39], separar [31], } \\
\text { separados [15], mamá [21], esperanza [14] }\end{array}$ \\
\hline Hijos (as) & $\begin{array}{l}\text { Preocupado [31], preocupan [37], extraño [27], extrañamos [47], fui } \\
\text { [21] }\end{array}$ \\
\hline
\end{tabular}

Fuente: elaboración propia.

Antes bien, uno de los campos conflictivos resulta ser el cuidado y el apoyo social a nivel familiar, que si bien son una de las dimensiones que influyen en la decisión de retornar, no siempre existe calidad, reciprocidad y apertura en muchas de las familias para la inclusión del miembro ausente, incluso en muchos de los casos, los viejos retornan a sus comunidades de origen en situación de aislamiento y soledad, aunque existe la expectativa de apoyo por parte de los viejos en las comunidades, lo cierto es que ellos han presenciado el alejamiento que genera la migración, sobre todo de aquellos que permanecieron en Estados Unidos muchos años haciendo complejo el intercambio y 
solidaridad intergeneracional o comunitaria (Guzmán, Huenchuán y Montes de Oca, 2003). Hablando de los vínculos comunitarios y sociales, las palabras que emergieron fueron adjetivos calificativos y sustantivos abstractos (buenas gentes, saludo, casa, raíces, amigos, querido, barato, espacio, tranquilo, nacimiento), lo que muestra el arraigo a las comunidades de origen, de igual forma que los morfemas verbales (quería, queríamos, quiero, vivir, vivo, cuidarnos, cuido, descansar) que expresan deseos y acciones concretas como en el caso del cuidado (cuido, cuidarnos) (tabla 8).

Tabla 8. Agentes comunitarios

\begin{tabular}{|l|l|}
\hline \multirow{3}{*}{ Gente } & $\begin{array}{l}\text { Piensan [18], pensé [35], Estados Unidos [58], linda [26], buenas } \\
\text { gentes [29], desconfianza [6], confianza [22], quería [18], queríamos } \\
\text { [21], quiero [17], pendiente [26], saludar [38], saludo [27], todos [35], } \\
\text { rodeados [17], cuide [37], tiempo [41], }\end{array}$ \\
\hline Pueblo & $\begin{array}{l}\text { Vivo [57], vivir [67], casa [101], ayudarnos [68], raíces [31], amigos } \\
\text { [42], querido [34], hambre [15], feliz [31], barato [17], descansar [69], } \\
\text { gana [41], mexicanos [67], México [48], tranquilo [45], pobrecillo [6], } \\
\text { nacimiento [47] }\end{array}$ \\
\hline
\end{tabular}

Fuente: elaboración propia.

De acuerdo con lo expuesto, el retorno se estructura desde ciertas palabras que narran esta experiencia. De ahí que en el lenguaje surjan no sólo las vivencias personales, sino también las relaciones de poder de las que han sido afectados. Por ello nos resultó importante rescatar dentro de las categorías aquellas palabras significativas a su representación del Estado y las instituciones, testimonios que evidencian falta de acciones y estrategias para ayudar al regreso de los connacionales. Particularmente se aprecia una preocupación por las instancias del sector salud, que si bien se reconoce que en México estos gastos son más accesibles, también se pone en tela de juicio la calidad y la eficiencia de los servicios proporcionados por las instituciones, sobre todo por las demandas en el primer y segundo nivel de atención médica para las personas mayores. Otro hecho que resulta ser indicativo de estas representaciones fue que durante las entrevistas se hace alusión a la falta de confianza en las instituciones (especialmente de aquellas encargadas de velar por la seguridad y la justicia), dado que al regreso algunos de ellos han sido víctimas de robos, extorciones y violencia (incluso en las Aduanas al momento de cruzar a México). Las personas mayores saben que en el país se vive un contexto de inseguridad, a pesar de ello intentan sobrellevar esas situaciones dentro de la familia y la comunidad. De modo que se aprecia una desconexión con otro tipo de instituciones (de asistencia social, por ejemplo). 
Tabla 9. Agentes políticos e institucionales

Enfermedad [41], checaron [21], medicamentos [32], doctor [26], confianza [39], gasto [18], confianza [22], violencia [9], desmadre [4], policías [17], ratero [12], gobierno [29]

Fuente: elaboración propia.

\section{CONCLUSIONES}

La migración como fenómeno social y lingüístico produce sujetos con una identidad en particular, misma que es expresada a través del lenguaje en cualquiera de sus manifestaciones. Estos juegos de significación de lo "otro" operan dentro de la conciencia de los sujetos haciendo que se creen a sí mismos, como también produzcan todo lo que les rodea, teniendo una posición concreta dentro de la realidad social. Es conveniente exponer que la migración opera bajo ciertos discursos que circulan en la cotidianidad, los cuales son aprehendidos por los sujetos, y en un acto de reflexividad, se incorporan a su conciencia. De tal suerte que en dentro de las tramas discursivas encontradas en este documento se destacan los arquetipos que hacen alusión a la comprensión del espacio (territorialidad) con distintas implicaciones para el bienestar de las personas mayores. Otros de los marcadores lingüísticos hacen referencia a los morfemas verbales, teniendo en cuenta que la acción implicada en ellos define al sujeto en un momento y espacio en particular. Finalmente, los agentes significativos juegan un papel esencial de la relación siempre dialéctica entre el migrante y los otros que le ayudan, por un lado, a definirse, y por el otro, a tener una posición en el mundo respecto a "los otros". En estos agentes pudimos percatarnos de la importancia que juega el cónyuge, la familia, los hijos e incluso Dios como mecanismo de contención ante la migración. Por lo que, más allá de conclusiones, consideramos que este documento abre nuevas vetas de análisis sobre el tema en cuestión y alienta a nuevos abordajes lingüísticos que permitan conocer las distintas implicaciones del lenguaje en la movilidad humana.

Una de las posturas que pretendimos problematizar en este trabajo, y que surge también del análisis de contenido, es situar el retorno de los mayores desde diferentes dimensiones de análisis. Claro está que es un asunto lingüístico que se inserta dentro de una cultura concreta, pero esa mirada en sí misma no particulariza (fragmenta/ aísla) que el regreso de los mayores se vuelve completo no sólo para la propia persona (dadas sus características legales, de salud, de redes sociales, de ingreso, etc.), sino que también es un llamado a posicionar este asunto desde la agenda política, que valga decir está invisibilizado dentro de las discusiones y programas sociales a favor del envejecimiento y la migración, a pesar de la importancia que ha adquirido en los últimos tiempos el regreso a las comunidades de origen. Esta 
visión no política está inserta dentro de los discursos de las personas mayores, pues en los acercamientos que tuvimos con ellos hacen referencia a otros agentes significativos (ellos mismos, la familia, la comunidad), pero que las acciones gubernamentales (tanto aquí como en Estados Unidos) no son narrativas frecuentes en ellos, podríamos decir que hay una cierta desconexión de sujeto con estas entidades políticas, ¿Qué significa esto?, una de las interpretaciones pudiera estar encaminada a que el migrante asume de manera privada e individual su regreso, prescindiendo de la participación que deben tener las instituciones y el Estado para asegurarle el cumplimiento de derechos humanos en esta etapa de vida, entonces desde estos discursos se aprecia una cultura de indefensión claramente difundida dentro de la cultura migratoria y afianzada por los tomadores de decisiones en ambos países.
Dentro de las entrevistas pudimos apreciar que el retornado mayor regresa a Durango con una expectativa hasta cierto punto humanizadora, pues se mueven a un futuro con sentido, que generalmente va acompañado de la huida del malestar (aislamiento, xenofobia, peligro, incertidumbre, presiones laborales, etcétera) que se viven como atentados contra la dignidad humana. Sin embargo, este regreso se vuelve conflictivo para el Estado y para las instituciones que no cuentan con la voluntad política y las capacidades (presupuestarias, programáticas, normativas) para dar atención a este fenómeno que, aunque hasta ahora no es masivo, irá incrementándose paulatinamente. Dicho esto, aquí la apuesta está en hacer del mayor retornado un sujeto político, concretamente es deber ético del Estado la generación de políticas públicas a favor de este grupo social con necesidades especiales a nivel nacional, estatal y local. 


\section{REFERENCIAS}

Ariza, M. (2002). Migración, familia y transnacionalidad en el contexto de la globalización: algunos puntos de reflexión. Revista Mexicana de Sociología, 64(4), 53-84.

Ávila, J. y Tuirán, R. (2000). Resultados del estudio binacional México-Estados Unidos sobre Migración. En: R. Tuirán. Migración México-Estados Unidos. Presente y futuro (pp. 66-87). Consejo Nacional de Población.

Bauman, Z. (2007). Identidad. Losada.

Berger P. y T. Luckmann. (2008). La construcción social de la realidad. Amorrortu.

Boivin M., Rosato A. y V. Arribas (1999). Constructores de la otredad. Una introducción a la Antropología social y Cultural. Eudeba.

Consejo Nacional de Evaluación de la Política de Desarrollo Social [CONEVAL]. (2020). Informe de pobreza y evaluación 2020. Durango. CONEVAL.

Consejo Nacional de Población (2010). Migración México-Estados Unidos. Panorama regional $y$ estatal. CONAPO

Consejo Nacional de Población. (2015a). Intensidad migratoria a nivel estatal y municipal. CONAPO.

Consejo Nacional de Población [CONAPO]. (2015b). El retorno en el nuevo escenario de la migración entre México y Estados Unidos. CONAPO.

Duque, E. (2014). Análisis de contenido mediante análisis de palabras clave. La representación de los participantes en los discursos de Esperanza Aguirre. Mediaciones Sociales, 13(1), 39-73. http://dx.doi.org/10.5209/ rev_MESO.2014.n13.49432

Flick. U. (2007). Introducción a la investigación cualitativa. Ediciones Morata y Fundación PAIDEIA GALIZA.

Foucault M. (2006). Las palabras y las cosas. Una arqueología de las ciencias sociales. Siglo Veintiuno Editores.

García Zamora, R. y Gaspar Olvera, S. (2016). Adultos mayores nacidos y residentes en México con vínculos migratorios internacionales (20002010). Revista de Estudios Migratorios, 3(5), 152-180.

Gergen, K. (1996). Realidades y relaciones. Aproximaciones a la construcción social. Paidos.

Gergen, K. (2006). El yo saturado. Dilemas de identidad en el mundo contemporáneo. Paidos.

Giorguli, S. y Bautista de León, A. (2018). Radiografía de la migración de retorno 2015. Colegio de México y CNDH. https://migracionderetorno. 
colmex.mx/wpcontent/uploads/2018/08/PB_1_radiografia_migracion_ retorno_2015.pdf

González González, G. (2009). Percepciones sociales sobre la migración en México y Estados Unidos: ¿Hay espacios para cooperar? En: L. Herrera Lasso. México, país de migración (pp. 107-150). Siglo XXI.

Guzmán, J., Huenchuan, S. y Montes de Oca, V. (2003). Redes de apoyo social de las personas mayores: marco conceptual. Notas de Población, 77(1).

Huerta Rosas, A. (2008). La construcción social de los sentimientos desde Pierre Bourdieu. Iberoforum, 3(5), 1-11.

Instituto Nacional de Estadística y Geografía [INEGI]. (2015). Encuesta Intercensal. INEGI.

Jáuregui Díaz, J. y Recaño Valverde, J. (2014). Una aproximación a als definiciones, tipoogicas y marcos teóricos de la migración de retorno. Revista Bibliográfica de Geografía y Ciencias Sociales, XIX(1084). http://www. ub.edu/geocrit/b3w-1084.htm

Jofré, J. L. (2001). Todas las otredades de la otredad. La construcción discursiva de la otredad en el acontecimiento del 11 de septiembre del 2001 . Time, una aproximación sociosemiotica, 5(10), 125-156.

López Castro, G. y L. Díaz Gómez (2018). Solo, viejo y sin amor. Migrantes de retorno y tercera edad en el bajío zamorano. En: N. Baca Tavira y A. Mojica Madrigal (coords.). Movilidades y migrantes internacionales. Reflexiones sobre los campos de relaciones socioeconómicas en comunidades migrantes en México y Estados Unidos. GEDISA.

López Silva, M. (2013). Realidades, construcciones y dilemas. Una revisión filosófica al construccionismo social. Universidad de A. Coruña.

Lozano, J., Peña-Marín, C. y Abril, G. (2009). Análisis del discurso. Hacia una semiótica de la interacción textual. Ediciones Cátedra.

Lújan Ponce, N. y Ramírez Sandoval, I. (2012). Experiencia migratoria en adultos mayores de Villa López, Chihuahua. En: M. D. París Pombo (coord.). Migrantes, desplazados, braceros y deportados. Experiencias migratorias y prácticas políticas (pp. 177-205). Colegio de la Frontera Norte, A.C.

Molina Foncerrada, M. (2009). Impactos económicos y sociales de la migración. En: L. Herrera Lasso. México, país de migración (pp. 157-168). Siglo XXI.

Montaño de la Concha, S. y Herrera Lasso, L. (2009). Los mexicanos en el mercado laboral estadounidense. En: L. Herrera Lasso. México, país de migración (pp. 19-72). Siglo XXI. 
Montes de Oca Zavala, V., Molina, A. y Avalos, R. (2009). Migración, redes trasnacionales y envejecimiento. Instituto de Investigaciones Sociales-UNAM.

Montoya Ortiz, M. y González Becerril, J. (2015). Evolución de la migración de retorno en México: migrantes procedentes de Estados Unidos en 1995 y de 1999 a 2014 . Papeles de población, 21 (85), 1 -18. http://www.scielo.org. $\mathrm{mx} /$ scielo.php?script $=$ sci_arttext\&pid $=$ S1 405-74252015000300003

Neimeyer, R. (1999). Narrative disruptions in the construction. En: R. A. Neimeyer y J. D. Raskin (Eds.), Constructions of disorder: Meaning-making frameworks for psychotherapy. American Psychological Association. https://doi.org/10.1037/10368-009

Nosetto, L. (2017). Las palabras y las cosas. Michael Foucault y la centralidad de la cuestión del origen de los discursos. Nómadas, 51 (2).

Osorio Campillo, H., Maya Sierra, T. y Rojas Sánchez, E. (2015). Territorios y migraciones. Territorialidades en transformación. Revista Bitácora Urbano Territorial, 25(1), 93-102.

Pérez Sales, P. y Lucena, R. (2000). Duelo: una perspectiva trascultural más allá del rito: la construcción social del sentimiento de dolor. Psiquiatría Pública, 12(3), 259-271.

Prospero, J. y V. Sieglin (2008). Migración, emociones y relaciones de poder. La danza ritual como espejo y medio de conflictos. Universidad Autónoma de Nuevo León y Plaza y Valdez editores.

Ramos Tovar, M. E. (2003). Entre tristeza y la esperanza: reconstrucciones identitarias de los mexicanos en Estados Unidos. En Ramos Tovar, M. E. (Coord.). Migración e identidad. Emociones, familia y cultura. Fondo Editorial de Nuevo León.

Reyes Reyes, L. (2016). Análisis de la migración hacia Estados Unidos de Américo, en la región norte de México. ru.iiec.unam.mx/3901/1/161-Reyes.pdf

Reyes Tovar, M. y Martínez Ruíz, D. T. (2015). La configuración identitaria en los territorios de migrantes internacionales. Península, 10(2), 117-133.

Ribeiro, M. (2002). Vejez, familia y política social. Perspectivas sociales/Social Perspectives, 4(1), 147-168.

Riosmena F., González M. y R. Wong (2012). El retorno reciente de Estados Unidos: salud, bienestar y vulnerabilidad de los adultos mayores. Coyunt Demogr., 2(1), 63-67. https://www.ncbi.nlm.nih.gov/pmc/articles/ PMC3578737/

Rivera Sánchez, L. (2013). Migración de retorno y experiencias de reinserción en la zona metropolitana de la Ciudad de México. Revista Interdisciplinaria de Mobilidad Humana, 41 (1), 55-76. 
Salas Astrain, R. (2012). Intersubjetividad, otredad y reconocimiento. Diálogos fenomenológicos para pensar la cuestión del otro en la filosofía intercultural. Cuadernos de la Facultad de Humanidades y Ciencias Sociales, 42, 83-104.

Secretaria de Gobernación [SEGOB]. (2017). Prontuario sobre migración mexicana de retorno. SEGOB.

Sobrino, J. (2013) Dinámica de la migración en México en la primera década del nuevo milenio. Instituto de Investigaciones Jurídicas de la UNAM.

Tuirán, R. (2000). La migración hacia Estados Unidos: respuestas gubernamentales. En: R. Tuirán. Migración México-Estados Unidos. Presente y futuro (pp. 22-44). Consejo Nacional de Población.

Zarur Osorio, A. (2018). Migración de retorno. Cuando regresas ves que nada ha cambiado, pero también te das cuenta que ya nada es igual. Historia de Mario. En: N. Baca Tavira y A. Mojica Madrigal (coords.). Movilidades y migrantes internacionales. Reflexiones sobre los campos de relaciones socioeconómicas en comunidades migrantes en México y Estados Unidos. GEDISA.

Zúñiga Hernández, E. y Gaspar, S. (2009). Migración y circularidad: el caso de México. En: L. Herrera Lasso, México, país de migración (pp. 206-228). Siglo XXI. 